\title{
Radiant-Heat Transfer Between Nongray Parallel Plates ${ }^{1}$
}

\author{
Stanley Goodman
}

\begin{abstract}
Radiant-heat transfer rates between infinite parallel plates were computed for Inconel and aluminum over a wide range of plate temperatures. Results indicate that assuming the plates to be graybodies introduces an error of 2 to 29 percent in the computed heattransfer rate.

In cross-sectional heat flow through box beams typical of aircraft structures, it was found that radiant-heat transfer predominates in Inconel beams and is significant in aluminum beams.
\end{abstract}

\section{Introduction}

The current need for predicting thermal stresses and deformations in aircraft structures gives rise to a need for determining the transient temperature distribution in these structures. As the temperatures in a structure increase, radiation becomes more important as a mode of heat transfer.

In computing radiant-heat transfer, a conventional assumption used is that the materials involved are graybodies, i. e., have spectral emissivities that are independent of wavelength. However, for polished metallic surfaces, spectral emissivities generally vary with wavelength, as is indicated by theoretical relationships for spectral emissivity of smooth-surfaced electrical conductors [1] ${ }^{2}$ and by published experimental data [2 to 7].

The purposes of this investigation were (1) to determine the magnitude of error caused by assuming that certain metals and alloys are graybodies in computing heat-transfer rates for a range of temperatures, temperature differences, and emissivities, and (2) to compare the radiant-heat transfer rate with the conductive-heat transfer rate in a box beam for a range of temperatures and temperature differences for two structural metals of different emissivities and thermal conductivities. Gaseous heat transfer is neglected.

\section{Symbols}

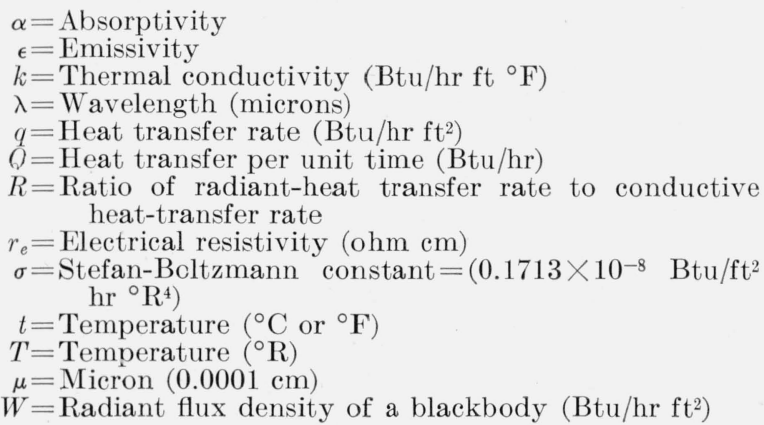

1 This work was conducted at the National Bureau of Standards under the sponsorship and with the financial assistance of the Office of Naval Research.

2 Figures in brackets indicate the literature references at the end of this paper.

\author{
SUBSCRIPTS \\ $b=$ Black body \\ $c=$ Conductive \\ $h=$ Hemispherical \\ $\lambda=$ Spectral \\ $0=$ Normal \\ $r=$ Radiant \\ $t=$ Total \\ $1=$ Hotter of two parallel surfaces involved in radiant \\ heat exchange \\ $2=$ Cooler of two parallel surfaces involved in radiant \\ heat exchange.
}

\section{Computations}

\subsection{Materials}

Computations were limited to appropriate materials for which the infrared spectral-emissivity curves are available in the literature. Such curves are presented in figure 1 for 99.6-percent-pure aluminum [7] and for Inconel [6].

\subsection{Structure}

The structure analyzed was a box beam with two opposite walls at different uniform temperatures. The other two walls were assumed to be perfect reflectors with identical temperature distributions. Two beams were considered, one relatively thinwalled and one relatively thick-walled (see sketch, table 1).

\section{Method of Analysis}

For internal radiant-heat transfer the box beam described is obviously equivalent to a pair of infinite parallel planes with temperatures equal to the boxbeam wall temperatures.

In order to cover the entire wave band over which significant radiant-energy exchange occurs, values of $\epsilon_{\lambda}$ beyond the experimental wavelength ranges [6 and 7] in figure 1 were obtained by extrapolation. The wave bands used in the computations were: for aluminum from $\lambda=0$ to $\lambda=25 \mu$, and for Inconel from $\lambda=0$ to $\lambda=15 \mu$. For all cases considered, at least 95 percent of the emitted radiant energy is within these wavelength ranges. The proportion of this emitted radiant energy within the wave bands in which the experimental values of $\epsilon$ were determined ranges roughly from 65 to 85 percent. 


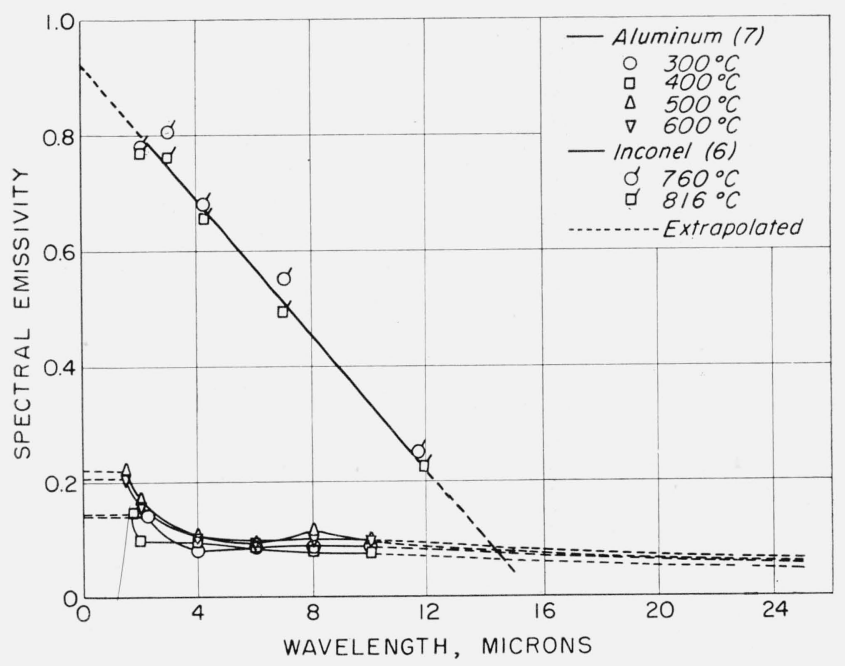

FIGURE 1. Spectral normal emissivities of polished aluminum and as-rolled Inconel.
To approximate the values of $\epsilon_{\lambda_{0}}$ outside the range of experimental determinations, $\epsilon_{\lambda_{0}}$ was extrapolated linearly for Inconel. For aluminum, $\epsilon_{\lambda_{0}}$ was taken as constant in the shorter wavelength range. (Relatively very little energy is emitted by the aluminum in this wavelength range.) For the longer wavelengths, $\epsilon_{\lambda_{0}}$ was assumed to vary inversely with $\sqrt{\lambda}$ [1]. The extrapolated values are shown in figure 1 .

\subsection{Computation of Radiant-Heat Transfer, Using Graybody Assumption}

In this case, the heat-transfer rate was computed from the formula given in reference [8] for net heat exchange between infinite parallel gray planes,

$$
q_{r}=\sigma\left(T_{1}^{4}-T_{2}^{4}\right) \frac{1}{1 / \epsilon_{1 t h}+1 / \epsilon_{2 t h}-1} .
$$

To compute $\epsilon_{t h}, \epsilon_{t 0}$ was first computed from the measured values of $\epsilon_{\lambda_{0}}$ for aluminum and Inconel (fig. 1), by use of the relationship

TABLE 1. Ratio, $R$, of radiant-heat transfer rate, $q_{r}$, to conductive-heat transfer rate, $q_{\boldsymbol{e}}$, for thin-walled and thick-walled beams

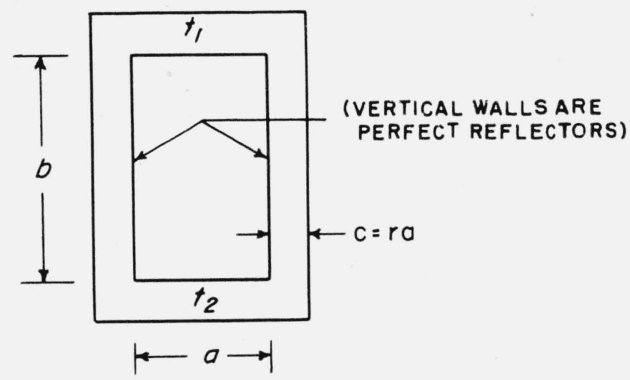

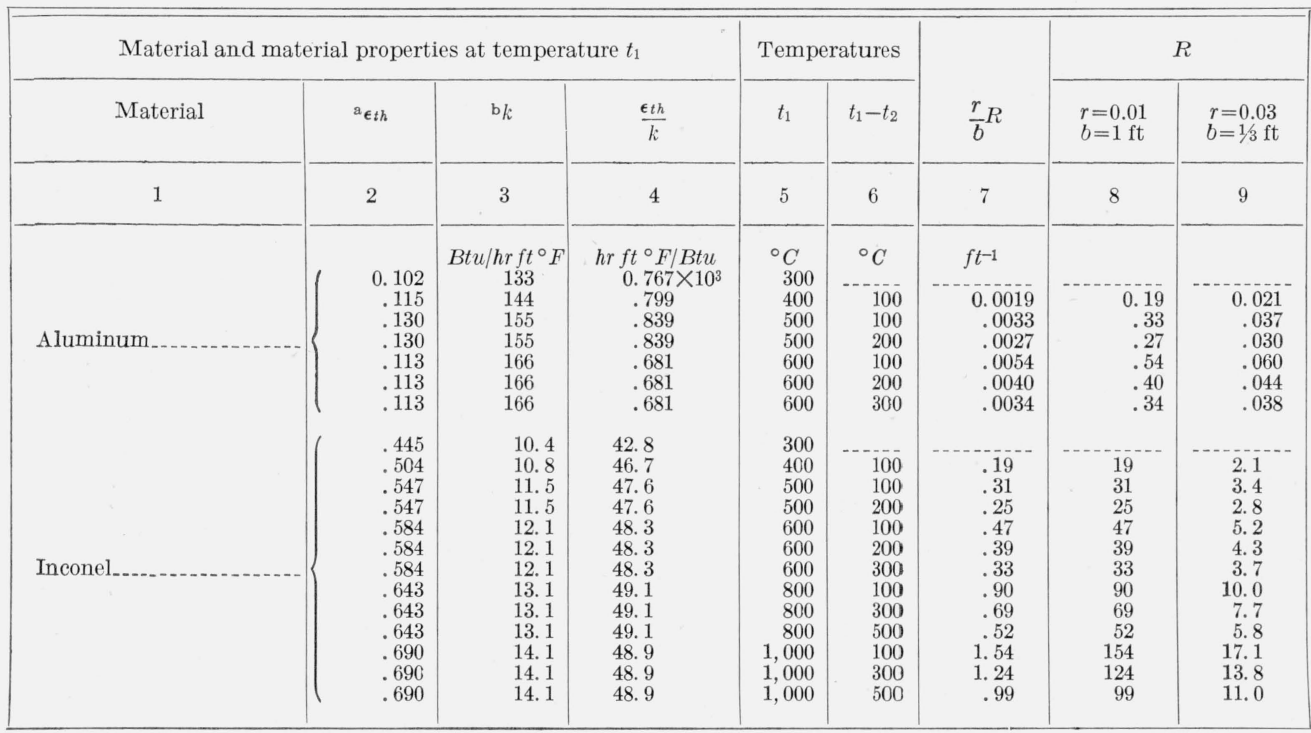

a Total hemispherical emissivity.

b Thermal conductivity. 


$$
\epsilon_{t 0}=\int_{0}^{\infty} \epsilon_{\lambda 0} \frac{W_{b \lambda 0}}{\frac{1}{\pi} \sigma T^{5}} d(\lambda T)=\int_{0}^{\infty} \epsilon_{\lambda 0} \frac{W_{b \lambda h}}{\sigma T^{5}} d(\lambda T) .
$$

Values of $W b \lambda h / \sigma T^{5}$ as a function of $\lambda T$ were taken from table 1 of reference [9] and substituted in eq (2), which was then integrated numerically. For aluminum, values of $\epsilon_{t h}$ were obtained from $\epsilon_{t 0}$ values by use of Schmidt and Eckert's relationship between $\epsilon_{t 0}$ and $\epsilon_{t h} / \epsilon_{t 0}$ for smooth-surfaced metals [1]. Inconel $\left(0.44<\epsilon_{t 0}<0.690\right)$, which forms an oxide surface layer on heating, was assumed to be intermediate between an electrical conductor and an insulator. The ratio $\epsilon_{t h} / \epsilon_{t 0}$ should lie between 0.95 and $1.05[1] ; \epsilon_{t h}$ was taken as equal to $\epsilon_{t 0}$. Appropriate values of $\epsilon_{t h}$ were substituted in eq (1), which was then solved for $g_{r}$.

\subsection{Computation of Radiant-Heat Transfer Rate From $\epsilon_{\lambda h}$}

Taking $\alpha_{\lambda h}=\epsilon_{\lambda h}$ and summing the series of reflections back and forth between planes 1 and 2 for the net radiant-heat transfer rate from plane 1 to plane 2 for monochromatic radiation, $g_{\lambda}$, gives

$$
\left.q_{\lambda}\left[\left(W_{b \lambda h}\right)_{T=T_{1}}-W_{b \lambda h}\right)_{T=T_{2}}\right] \frac{1}{1 / \epsilon_{1 \lambda h}+1 / \epsilon_{2 \lambda h}-1} .
$$

For aluminum, $\epsilon_{\lambda h}$ was computed from Schmidt and Eckert's relationships between $\epsilon_{\lambda_{0}}$ and $r_{e} / \lambda$, and between $r_{e} / \lambda$ and $\epsilon_{\lambda h}$ [1]. Values of $W_{b \lambda h}$ were computed from table 1 of [9]. For Inconel, because $\epsilon_{t h} \approx \epsilon_{t 0}$, it follows that $\epsilon_{\lambda h} \approx \epsilon_{\lambda 0}$, and $\epsilon_{\lambda h}$ was taken as equal to $\epsilon_{\lambda_{0}}$.

To compute the radiant-heat transfer rate between planes 1 and 2 , the appropriate values of $W_{b \lambda h}$ and $\epsilon_{\lambda h}$ were substituted in eq (3) and the equation was then integrated numerically over the wave band $\lambda=0$ to $\lambda=25 \mu$ for aluminum and $\lambda=0$ to $\lambda=15 \mu$ for Inconel.

\subsection{Computation of Conductive-Heat Transfer Rate}

Walls 1 and 2 of the box beam were assumed to have uniform temperature distributions. The heat transfer per hour was computed from

$$
Q_{c}=k A \frac{\left(t_{1}-t_{2}\right)}{b}
$$

where

$Q_{c}=$ conductive-heat transfer per unit time (Btu/hr),

$A=$ total cross-sectional area of conducting walls $\left(\mathrm{ft}^{2}\right)$,

$b=$ distance between radiating wall surfaces (ft).

Values of $k$, taken from reference [8] for aluminum and from reference [10] for Inconel, are listed in column 3 of table 1 . The values of $k$ used in eq (4) are taken at the temperature $1 / 2\left(t_{1}+t_{2}\right)$.

Values of the ratio $R=Q_{\tau} / Q_{c}=q_{\tau} / q_{c}$ were used as a measure of the relative importance of radiant and conductive heat transfer in a box beam:

$$
R=\frac{Q_{r}}{Q_{c}}=\frac{q_{r} l a}{k 2 c l \frac{t_{1}-t_{2}}{b}} \frac{q_{r}}{2 k\left(t_{1}-t_{2}\right)} \frac{b}{r},
$$

where

$$
\begin{aligned}
l & =\text { length of beam }(\mathrm{ft}), \\
a & =\text { width of radiating surface }(\mathrm{ft}), \\
c=r a & =\text { thickness of each conducting wall }(\mathrm{ft}), \\
b & =\text { distance between radiating wall surfaces } \\
& (\mathrm{ft}) .
\end{aligned}
$$

\section{Results}

Values of $q_{r}$ computed from the actual spectral emissivities, values of $q_{r}$ computed from total emis-

\begin{tabular}{|c|c|c|c|c|c|}
\hline Material & $t_{1}$ & $t_{1}-t_{2}$ & ${ }^{a} q_{r}$ & ${ }^{b} q_{r}$ & $\frac{{ }^{a} q_{r}-{ }^{b} q_{r}}{{ }^{a} q_{r}}$ \\
\hline 1 & 2 & 3 & 4 & 5 & 6 \\
\hline Aluminum & $\begin{array}{l}{ }^{\circ} \mathrm{C} \\
400 \\
500 \\
500 \\
600 \\
600 \\
600\end{array}$ & $\begin{array}{l}{ }^{\circ} C \\
100 \\
100 \\
200 \\
100 \\
200 \\
300\end{array}$ & $\begin{array}{c}\text { Btu/hr f } t^{2} \\
97 \\
178 \\
283 \\
311 \\
451 \\
548\end{array}$ & $\begin{array}{c}\text { Btu/hr f } t^{2} \\
95 \\
157 \\
271 \\
259 \\
385 \\
483\end{array}$ & $\begin{array}{r}\% \\
2 \\
12 \\
4 \\
17 \\
15 \\
12\end{array}$ \\
\hline Inconel & $\begin{array}{r}400 \\
500 \\
500 \\
600 \\
600 \\
600 \\
800 \\
800 \\
800 \\
1,000 \\
1,000 \\
1,000\end{array}$ & $\begin{array}{l}100 \\
100 \\
200 \\
100 \\
200 \\
300 \\
100 \\
300 \\
500 \\
100 \\
300 \\
500\end{array}$ & $\begin{array}{r}726 \\
1,250 \\
1,980 \\
1,980 \\
3,240 \\
3,970 \\
4,140 \\
9,110 \\
11,090 \\
7,660 \\
17,900 \\
22,800\end{array}$ & $\begin{array}{r}543 \\
973 \\
1,460 \\
1,590 \\
2,510 \\
2,880 \\
3,540 \\
7,320 \\
7,820 \\
6,780 \\
15,000 \\
17,900\end{array}$ & $\begin{array}{l}25 \\
22 \\
26 \\
20 \\
23 \\
27 \\
15 \\
24 \\
29 \\
12 \\
16 \\
21\end{array}$ \\
\hline
\end{tabular}
sivities, using a graybody assumption, and the percentage difference between corresponding values are listed in columns 4,5 , and 6 , respectively, of table 2 .

The high temperatures for aluminum were intended to give an upper limit for the effect of radiantheat transfer.

TABLE 2. Computed radiant-heat flow rate, $q_{r}$, between infinite parallel plates at temperatures $t_{1}$ and $t_{2}$

a Computed from spectral emissivity. $b$ Computed from total emissivity.

Ratios of radiant-heat transfer rate to conductiveheat transfer rate are listed in table 1 for a box beam in which all radiant-heat transfer is between the horizontal walls, and all conductive-heat transfer is in the vertical walls. Column 7 of the table lists ratios of radiant-heat transfer to conductive-heat transfer, $R$, times dimension ratio, $r / b$, where $r=c / a$ is the ratio of vertical-wall thickness to horizontalwall width, and $b$ is the distance between horizontalwall surfaces. Values of $R$ for a box beam with $c / a=0.01$ and $b=1 \mathrm{ft}$ are listed in column 8 of table 1 , and values of $R$ for a box beam with $c / a=0.03$ and $b=1 / 3 \mathrm{ft}$ are listed in column 9 . 


\section{Discussion}

The shapes of the spectral-emissivity curves (see fig. 1) for aluminum and Inconel differ widely; for Inconel the curve decreases linearly with increasing wavelength. DeCorso and Coit $[6]$ state that the spectral emissivity of Inconel should not vary with temperature if the surface condition is maintained. For aluminum the curve approaches graybody emissivity for $\lambda>2.5 \mu$, and is temperature dependent. The effect of $t_{1},\left(t_{1}-t_{2}\right)$, and $\epsilon$ on the size of the error that is caused by assuming the materials are graybodies when computing radiant-heat flow rates is dependent on the shape of the spectral-emissivity curve. For aluminum the error increases with increasing $t_{1}$ and with decreasing $\left(t_{1}-t_{2}\right)$. For Inconel, with greater $\epsilon_{\lambda}$ (and therefore $\alpha_{\lambda}$ ) than aluminum, the error is greater than in aluminum and increases with decreasing $t_{1}$ and with increasing $\left(t_{1}-t_{2}\right)$.

For aluminum and those materials that approach graybodies for the longer wavelengths, the graybody assumption may be used when the proportion of energy radiated at the shorter wavelengths is small. Pure aluminum appears to act like a graybody when its radiating surfaces are at temperatures lower than $400^{\circ}$ C. Due to the paucity of spectral-emissivity data, it is not known whether or not pure aluminum and Inconel have $\epsilon_{\lambda}$ curves typical of other aircraft structural materials.

The total emissivity of a material depends more on its surface condition, history of heating, oxidation, cleanliness of surface, and wetness than on its chemical composition. For example, Hase [7] gives the following values of $\epsilon_{\lambda}$ (and therefore $\alpha_{\lambda}$ ) for pure aluminum at $400^{\circ} \mathrm{C}$ for various surface conditions at $\lambda=2$ : Sandblasted, 0.63; dull, 0.29; polished, 0.17. For Inconel, DeCorso and Coit [6] give the following values of $\epsilon_{t}$ at $1,000^{\circ} \mathrm{F}$ after various periods of heating: Initial, 0.28 ; after $15 \mathrm{~min}$ at $1,500^{\circ} \mathrm{F}, 0.53$; after $30 \mathrm{~min}$ at $1,800^{\circ} \mathrm{F}, 0.62$; after $135 \mathrm{~min}$ at $2,000^{\circ}$ $\mathrm{F}, 0.80$. It follows that for the cases considered, the error in predicting $q_{r}$ due to the error in the value of $\epsilon$ may be greater than the error due to use of a graybody assumption for nongray materials.

The beams chosen for analysis are common to many aircraft structures. Radiant-heat transfer will have its greatest value for this type of geometrical configuration and will be independent of the distance between the radiating walls. Because the quantity of energy radiated from a body varies as the fourth power of the absolute temperature of the body, whereas the quantity of energy conducted in a body depends on the temperature gradient, the ratio of radiant- to conductive-heat transfer rate, $q_{r} / q_{c}$, must be greatest for high temperatures. For box beams of the same geometry, $q_{r} / q_{c}$ must increase with increasing $\epsilon / k$. The large differences between the $q_{r} / q_{c}$ values for corresponding wall temperatures in aluminum and Inconel are due to the fact that $\epsilon / k$ is comparatively small for aluminum and large for Inconel. With increasing temperature, $\epsilon_{t h} / k$ generally increases in the reasonable structure temperature range (see table 1).

For a given structure, the size of the error in com- puted temperature distribution due to error in the computed radiant-heat transfer rate depends on the proportion of radiant-heat transfer to total heat transfer. It would be greater for Inconel, with a large $\epsilon / k$ ratio, than for aluminum, with a small $\epsilon / k$ ratio.

\section{Conclusions}

The error due to assuming pure aluminum and Inconel are graybodies in computing the radiantheat transfer between infinite parallel plates of these materials over a wide range of temperatures and temperature differences varies from 2 to 29 percent. An error of this magnitude might also be encountered in determining the emissivity of a given material, because of the large variation in $\epsilon$ with surface conditions. The size of the error for given plate temperatures and emissivities depends on the shape of the spectral-emissivity curve. Lack of spectralemissivity data makes it difficult to determine the error for other aircraft materials.

There is a need for determination of spectral hemispherical emissivities of aircraft materials at various temperatures and for various surface conditions.

The ratio of radiant-heat transfer to conductiveheat transfer in a pure aluminum box beam (in the absence of gaseous heat transfer) ranges from 0.19 to 0.54 for a thin-walled beam and from 0.021 to 0.06 for a thick-walled beam over a range of hotwall temperatures from $400^{\circ}$ to $600^{\circ} \mathrm{C}$ and a range of wall-temperature differences from $100^{\circ}$ to $300^{\circ} \mathrm{C}$. For an Inconel box beam the corresponding ratios are: thin-walled, 19 and 154; thick-walled, 2.10 and 17.1 , over a range of hot-wall temperatures from $400^{\circ}$ to $1,000^{\circ} \mathrm{C}$ and a range of wall-temperature differences of $100^{\circ}$ to $500^{\circ} \mathrm{C}$. These results for a particular box beam indicate that radiant-heat transfer predominates for Inconel structures and is significant for thin-walled aluminum structures, at elevated temperatures.

\section{References}

[1] M. Jakob, Heat transfer, vol. 1, 1st ed., p. 44-52 (John Wiley \& Sons, Inc., New York, N. Y., 1949).

[2] Von W. Sieber, Zusammensetzung der von Werk-und Baustoffen zuruckgeworfenen Warmestrahlung, Z. tech. Phys. 22, 130 (1941).

[3] E. Hagan and H. Rubens, Das Reflexionsvermogen von Metallen und belegten Glasspiegeln, Ann. Physik 1, 352 (1900).

[4] D. J. Price, The emissivity of hot metals in the infrared, Proc. Phys. Soc. London 59 (pt. 1), 118 (1947).

[5] W. E. Forsythe and E. Q. Adams, Radiating characteristics of tungsten and tungsten lamps, J. Opt. Soc. Amer. 35, 108 (1945).

[6] S. M. DeCorso and R. L. Coit, Measurement of total emissivities of gas-turbine combustor materials, Paper 54-SA-26, Am. Soc. Mech. Engrs., Pittsburgh, Pa., meeting (June 20-24, 1954).

[7] R. Hase, Emission von Aluminium und seinen Legierungen, Z. tech. Phys. 13, 145 (1932).

[8] W. H. McAdams, Heat transmission, 3d ed., p. 63 (McGraw-Hill Book Co., Inc., New York, N. Y., 1954).

[9] R. V. Dunkle, Thermal radiation tables and applications, Trans. Am. Soc. Mech. Angrs. \%6, 549 (1954).

[10] Engineering properties of Inconel, Tech. Bul. T-7, p.3, International Nickel Co., New York, N. Y. (March 1950).

Washington, May 11, 1956. 